\title{
MODELLING EFFECTS OF HCV PLUS-STRAND RNA INFLUX INTO A CELL DURING HCV REPLICATION
}

\author{
Adamu Ishaku ${ }^{1,2}$, Nafiu Hussaini ${ }^{*, 2}$ \\ ${ }^{1}$ Department of Mathematics, Faculty of Sciences, Gombe State University, \\ Gombe, Gombe State, Nigeria. \\ adammaths@gsu.edu.ng \\ ${ }^{2}$ Department of Mathematical Sciences, Faculty of Sciences, Bayero \\ University, Kano, Kano State, Nigeria., \\ * Corresponding author: nhussaini.mth@buk.edu.ng
}

\begin{abstract}
This paper presents a subgenomic Hepatitis C Virus (HCV) replication model which incorporates the rate of influx of HCV plus-strand RNA into Huh-7 cell and monitored its effects. The model exhibits three equilibria, namely: trivial equilibrium, healthy equilibrium and endemic equilibrium. Stability analysis of the model reveals that the healthy equilibrium is globally asymptotically stable under certain condition. Furthermore, it is shown that increase in the rate of influx, increases the steady state level of total plus strand RNA, synthesized plus strand RNA, replicated plus strand RNA and NS5B in the system. Sensitivity and uncertainty analyses of the model (using the basic replication number $\left(\mathcal{R}_{0}\right)$ as the response function) show that the top three PRCC-ranked parameters are the rate of influx, $k_{0}$ of $\mathrm{HCV}$ plus-strand RNA, the rate of production of translation complex $\left(T_{c}\right)$ and the rate of degradation, $\mu_{p}^{c y t}$, of plus-strand RNA $R_{P}^{c y t}$. Furthermore, the distribution of $\mathcal{R}_{0}$ is between $[0.9999,1.0008]$ with a mean of $\mathcal{R}_{0}=1.0003$.

Key words and Phrases: Hepatitis C Virus, replication, basic replication number, stability analysis.
\end{abstract}

\section{INTRODUCTION}

Hepatitis C Virus (HCV) is a plus-strand RNA virus that attacks liver cells and causes liver failure which can lead to liver transplantation or death [3]. HCV is often asymptomatic, only in about $15 \%$ of the cases symptoms includes lost of

2020 Mathematics Subject Classification: 4811

Received: 03-06-2018, accepted: 19-03-2019. 
appetite, fatigue, nausea, muscles or joint pain, weight lost, etc. [3]. The virus is very small in size (about 30-60nm) [1], envelopes and belongs to the genus Hepacivirus in the family Flaviviridae [2]. The major route of transmitting this virus is via blood (i.e, during blood transfusion, use of poorly sterilized medical equipments that carried infected blood, etc) $[3,17]$. According to WHO, about 71 million persons are infected with HCV globally and among which about $15-45 \%$ clear the virus within 6 months. Approximately, 399,000 individuals die every year [30].

In order understand the dynamics, prevent and control the spread of HCV within an individual (in vivo) or in a population, several mathematical models have been proposed and rigorously analysed by different authors (see, for instance, $[2,3,8,14,25,26]$ and references therein). Spiegelman in 1970 [4] has shown that viral RNA amplification depends on RNA polymerase-containing RNA replicase that specifically interacts with the incoming viral RNA (plus-strand). In 1981, Biebricher et al [19] used the idea developed in [4] and studied the kinetics of RNA amplification by $Q \beta$ replicase (quantitatively) and developed a kinetic model for self-replication of $Q \beta$ RNA in vitro [5].

A model for complete life cycle of $Q \beta$ has been presented in [18]. Using a subgenomic genotype 1b (Con1) replicon, Lohmann et al. in [6] found that HCV RNA replication persist in a human hepatoma cell line (Huh-7)1 . Later, the adaptive mutations in nonstructural proteins were identified by Bartenschlager et al. in [7] which improved the efficiency of HCV replication. In 2007, Dahari et al. in [2] produced a model for complete replication of HCV. In this study, the model presented by Dahari et al. in [2] is extended by incorporating the rate of influx of $\mathrm{HCV}$ plus-strand into a cell and monitored (mathematically) its effects to the production of more HCV particles.

This paper is organized as follows. The model is formulated in Section 2. The model analysis is presented in Section 3. Numerical simulations of the extended model are given in Section 4. Sensitivity and uncertainty analyses are performed in Section 5.

\section{MODEL FORMULATION}

The model is derived from the details of HCV replication life cycle as described by Dahari et al [2] and other literature in [13, 15, 17]. We sub-divide the process into three stages viz: HCV entry, HCV translation, and HCV replication. Stage I: Hepatitis C Virus Entry

The first step of interaction between $\mathrm{HCV}$ and the target cell that is required for the initiation of the infection [9]. Recent studies of HCV entry revealed that the process is slow and complex multistep [11]. The virus cross the plasma membrane of the target cell and have access to cytosolic and/or nuclear components in order to replicate its genome [10]. The viral entry begins with binding of the HCV particle to an attachment factor on the cell surface [11]. After binding of the virus to the 
cell surface, the virus enters the cell by clathrin-dependent endocytosis and upon acidification, fusion of the viral envelope, presumably with the membrane of an early endosome will lead to the release of the viral nucleocapsid into the cytoplasm $[12]$.

Stage II: Translation of Hepatitis C Virus RNA

The plus-strand RNA $\left(R_{p}^{c y p}\right)$ enters the cell, it interacts with the host ribosome complex $\left(R_{i b o}\right)$ to produce translation complex $\left(T_{c}\right)$ at a constant rate $k_{1}$. Once this translation complex is being produced, then translation begins and viral polyprotein $(\mathrm{P})$ will be formed at a constant rate $k_{2}$. Thus, $T_{c}$ degrades at a constant rate $\mu_{T_{c}}$. After viral polyprotein is produced, we assume that the ribosome complex dissociate from $T_{c}$ and that will result to free plus-strand RNA. This $R_{p}^{c y p}$ degrades at the rate of $\mu_{p}^{c y t}$. Therefore, $R_{p}^{c y t}$ and $R_{i b o}$ disappear at a rate of $k_{1}$ and then reappear at a rate $k_{2}$. The viral polyprotein alone will then split at a constant rate $k_{c}$ into separate structural and non-structural viral protein. It is this nonstructural viral polyprotein that include NS5B polymerase $\left(E^{c y t}\right)$ which contains RNA replicase which is the replication machinery. Then, $E^{c y t}$ will then degrades with rate constant $\mu_{E}^{c y t}$. When NS5B is formed, then the translation is complete $[2]$.

Stage III: Replication of Hepatitis C Virus RNA

The non-structural viral protein (NS5B polymerase) enters the VMS at a constant rate $k_{E i n}$. Also, the free plus-strand that dissociate from $T_{c}$ will also enter the VMS at a constant rate $k_{\text {pin }}$. The free plus-strand in VMS $\left(R_{p}\right)$ will interact with NS5B polymerase in VMS $(E)$ to form plus-strand replicative intermediate complex $\left(R_{I p}\right)$ at a constant rate $k_{3}$. The produced $R_{I p}$ degrades at a rate $\mu_{I p}$. The complimentary minus-strand will then be formed at a constant rate of $k_{4 m}$ and the $R_{I p}$ dissociates to double strand RNA $\left(R_{d s}\right)$ and NS5B polymerase $(E)$. After the formation of $R_{d s}$, then $E$ will act on it and leads to the formation of double strand replicative intermediate RNA $\left(R_{I d s}\right)$ at a constant rate of $k_{5}$ and the formed $R_{I d s}$ degrades at a constant rate of $\mu_{I d s}$. The formed $R_{I d s}$ produces the replicate nascent plus-strand RNA at a constant rate of $k_{4 p}$ per complex and once this nascent plusstrand RNA is replicated. The unwounded plus-strand RNA $R_{p}$ is then released from $R_{I d s}$ complex (along with $R_{d s}$ and $E$ ). The replicated plus-strand RNA $\left(R_{P}\right)$ will degrade at a constant rate of $\mu_{p}$ and then transported back to the cytoplasm at a constant rate of $k_{\text {pout }}$. Figure 1 represents the diagrammatic explanation of HCV plus-strand RNA entry, translation and replication processes. The first four equations of the model (1) describe the translation process of $\mathrm{HCV}$, while the last five equations describe replication process of $\mathrm{HCV}$ as shown in Figure 1. 


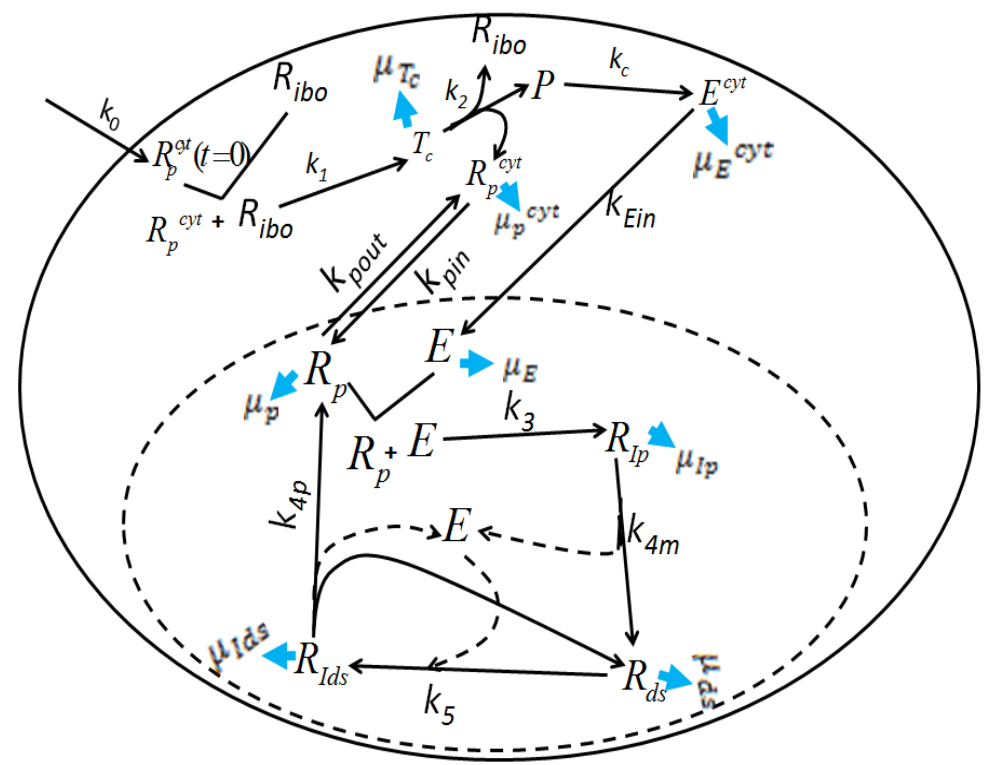

Figure 1. The Reaction Scheme for HCV Replication Process in Huh-7 Cell with $k_{0}$ as Rate of Influx of HCV Plus-strand RNA. The Cell Membrane is Symbolised by a Solid Oval Shape and VMS is Symbolised by Dotted Oval Shape.

$$
\begin{aligned}
\frac{d R_{p}^{c y t}}{d t} & =k_{0} R_{p}^{c y t}+k_{2} T_{c}+k_{\text {pout }} R_{p}-k_{1} R_{i b o} R_{p}^{c y t}-k_{p i n} R_{p}^{c y t}-\mu_{p}^{c y t} R_{p}^{c y t} \\
\frac{d T_{c}}{d t} & =k_{1} R_{i b o} R_{p}^{c y t}-k_{2} T_{c}-\mu_{T_{c}} T_{c} \\
\frac{d P}{d t} & =k_{2} T_{c}-k_{c} p \\
\frac{d E^{c y t}}{d t} & =k_{c} P-k_{E i n} E^{c y t}-\mu_{E}^{c y t} E^{c y t} \\
\frac{d R_{p}}{d t} & =k_{4 p} R_{I d s}+k_{p i n} R_{p}^{c y t}-k_{3} R_{p} E-k_{p o u t} R_{p}-\mu_{p} R_{p} \\
\frac{d R_{d s}}{d t} & =k_{4 m} R_{I p}+k_{4 p} R_{I d s}-k_{5} R_{d s} E-\mu_{d s} R_{d s} \\
\frac{d E}{d t} & =k_{E i n} E^{c y t}+k_{4 m} R_{I p}+k_{4 p} R_{I d s}-k_{3} R_{p} E-k_{5} R_{d s} E-\mu_{E} E \\
\frac{d R_{I p}}{d t} & =k_{3} R_{p} E-k_{4 m} R_{I p}-\mu_{I p} R_{I p} \\
\frac{d R_{I d s}}{d t} & =k_{5} R_{d s} E-k_{4 p} R_{I d s}-\mu_{I d s} R_{I d s}
\end{aligned}
$$


with the initial conditions

$$
\begin{aligned}
R_{p}^{c y t}(0) & \geq 0, \quad T_{c}(0) \geq 0, \quad P \geq 0, \quad E^{c y t} \geq 0, \quad R_{p} \geq 0 \\
R_{d s} & \geq 0, \quad E \geq 0, \quad R_{I p} \geq 0, \quad R_{I d s} \geq 0 .
\end{aligned}
$$

\begin{tabular}{|l|l|r|l|}
\hline Parameter & Description & Range/value & Citation \\
\hline$k_{1}$ & Rate of $T_{c}$ formation & $34-83$ & {$[2]$} \\
$k_{2}$ & polyprotein translation rate & 100 & Assumed \\
$k_{3}$ & $R_{I p}$ formation rate & $0.01-0.02$ & Assumed \\
$k_{4 p}$ & $R_{P}$ synthesis rate & 1.7 & Assumed \\
$k_{4 m}$ & $R_{d s}$ synthesis rate & 1.7 & Assumed \\
$k_{5}$ & $R_{I d s}$ formation rate & molecule $e^{-1}$ & {$[2]$} \\
$k_{\text {pout }}$ & Rate of transporting $R_{P}$ into cytoplasm & 0.2 & {$[2]$} \\
$k_{\text {pin }}$ & Rate of transporting $R_{P}^{\text {cht }}$ into VMS & 0.2 & {$[2]$} \\
$k_{E \text { in }}$ & Rate of transporting $E^{c y t}$ into VMS & $4 \times 10^{-6}-4 \times 10^{-5}$ & {$[2]$} \\
$k_{c}$ & Viral polyprotein cleavage rate & $0.2-1$ & {$[2]$} \\
$\mu_{p}^{c y t}$ & $R_{P}^{c y t}$ degradation rate & $0.06-15$ & Assumed \\
$\mu_{p}$ & $R_{P}$ degradation rate & 0.07 & {$[22]$} \\
$\mu_{d s}$ & $R_{d s}$ degradation rate & 0.06 & {$[23]$} \\
$\mu_{I p}$ & $R_{I p}$ degradation rate & $0.01-0.06$ & Assumed \\
$\mu_{I d s}$ & $R_{I d s}$ degradation rate & 0.13 & {$[2]$} \\
$\mu_{T_{c}}$ & $T_{c}$ degradation rate & $0.001-0.02$ & Assumed \\
$\mu_{E}$ & E degradation rate & $0.001-0.06$ & Assumed \\
$\mu_{E}^{c y t}$ & $E^{c y t}$ degradation rate & 0.06 & {$[23]$} \\
$R_{P}^{c y p}(0)$ & Initial quantity of plus-strand RNA & 500 & {$[2]$} \\
$R_{i b o}^{T o t}$ & Number of ribosome complex & 700 & {$[2]$} \\
\hline
\end{tabular}

TABLE 1. Parameter description and values for model (2) model 


\section{MODEL ANALYSIS}

For simplicity, let $R_{p}^{c y t}=x_{1}, T_{c}=x_{2}, P=x_{3}, E^{C y t}=x_{4}, R_{p}=x_{5}$, $R_{d s}=x_{6}, E=x_{7}, R_{I p}=x_{8}$, and $R_{I d s}=x_{9}$ in equation (1), we have:

$$
\begin{aligned}
\frac{d x_{1}}{d t} & =k_{0} x_{1}+k_{2} x_{2}+k_{\text {pout }} x_{5}-k_{1} R_{i b o} x_{1}-k_{\text {pin }} x_{1}-\mu_{p}^{c y t} x_{1} \\
\frac{d x_{2}}{d t} & =k_{1} R_{i b o} x_{1}-k_{2} x_{2}-\mu_{T_{c}} x_{2} \\
\frac{d x_{3}}{d t} & =k_{2} x_{2}-k_{c} x_{3} \\
\frac{d x_{4}}{d t} & =k_{c} x_{3}-k_{E i n} x_{4}-\mu_{E}^{c y t} x_{4} \\
\frac{d x_{5}}{d t} & =k_{4 p} x_{9}+k_{p i n} x_{1}-k_{3} x_{5} x_{7}-k_{\text {pout }} x_{5}-\mu_{p} x_{5} \\
\frac{d x_{6}}{d t} & =k_{4 m} x_{8}+k_{4 p} x_{9}-k_{5} x_{6} x_{7}-\mu_{d s} x_{6} \\
\frac{d x_{7}}{d t} & =k_{E i n} x_{4}+k_{4 m} x_{8}+k_{4 p} x_{9}-k_{3} x_{5} x_{7}-k_{5} x_{6} x_{7}-\mu_{E} x_{7} \\
\frac{d x_{8}}{d t} & =k_{3} x_{5} x_{7}-k_{4 m} x_{8}-\mu_{I p} x_{8} \\
\frac{d x_{9}}{d t} & =k_{5} x_{6} x_{7}-k_{4 p} x_{9}-\mu_{I d s} x_{9}
\end{aligned}
$$

with the initial conditions

$$
x_{i}(0) \geq 0, \quad i=1,2,3, \ldots, 9 .
$$

3.1. Positivity of solution. Since the model monitors number of molecules of replication mechanism during HCV replication, then we need to show that for nonnegative initial conditions, the variables $x_{i}(t), \quad i=1,2, \ldots, 9$ are positive and remain positive for all $t \geq 0$. Hence the following result.

Lemma 3.1. If $x_{i}(0)>0$, for $i=1,2, \ldots, 9$, then the solution $x_{i}(t)$, for $i=$ $1,2, \ldots, 9$ of the model (2) are positive for all $t \geq 0$.

Proof. Adopting the prove by contradiction method used in proving the positivity of solution of system of nonlinear equations in [27], we prove this lemma by contradiction.

Suppose there exists a time $t_{j}$ such that

$$
\begin{aligned}
& x_{j}\left(t_{j}\right)=0, x_{j}^{\prime}\left(t_{j}\right)<0, \quad j=1,2,3, \ldots, 9, \\
& x_{i}(t)>0 \quad i \neq j, \quad i=1,2,3, \ldots, 9, \quad 0<t<t_{j} .
\end{aligned}
$$

Since the model parameters are positive, then from assumption (3) and : first equation in model(2) (i.e $\mathrm{j}=1$ ), we have

$$
x_{1}^{\prime}\left(t_{1}\right)=k_{2} x_{2}+k_{\text {pout }} x_{5}>0,
$$


second equation in $\operatorname{model}(2)$ (i.e $\mathrm{j}=2)$, we have

$$
x_{2}^{\prime}\left(t_{2}\right)=k_{1} R_{i b o} x_{1}>0,
$$

third equation in model (2) (i.e $\mathrm{j}=3$ ), we have

$$
x_{3}^{\prime}\left(t_{3}\right)=k_{2} x_{2}>0
$$

fourth equation in model (2) (i.e $\mathrm{j}=4$ ), we have

$$
x_{4}^{\prime}\left(t_{4}\right)=k_{c} x_{3}>0,
$$

It is easy to show from assumption (3) and fifth, sixth, seventh, eighth, and ninth equations. Hence, the solutions of the model $(2), x_{j}(t)>0, \quad j=1,2,3,4,5,6,7,8,9$ remain positive for $t \geq 0$.

Now setting the right-hand side of model (2) to zero (noting that $R_{i b o}=$ $\left.R_{i b o}^{T o t}-x_{2}\right)$.

The following equilibria are identified.

(1) Trivial equilibrium i.e $E_{0}=(0,0,0,0,0,0,0,0,0)$.

(2) Healthy equilibrium i.e when a cell is healthy ( plus-strand RNA (which is $x_{1}$ ) is not in the cell and all other mechanisms (except $T_{c}$ ) are not produced and all other parameters are zeros ), thus $x_{1}=x_{3}=x_{4}=x_{5}=x_{6}=x_{7}=$ $x_{8}=x_{9}=0$. The number of free ribosome complexes involved in HCV RNA translation is calculated in [2] as $R_{i b o}=R_{i b o}^{T o t}-T_{c}$, then we say $T_{c}=R_{i b o}^{T o t}-R_{i b o}$. Let $T_{c}=\eta$, then $x_{2}=\eta$ where $\eta=R_{i b o}^{T o t}-R_{i b o}$, hence healthy equilibrium point $E_{1}=(0, \eta, 0,0,0,0,0,0,0)$.

(3) Endemic equilibrium i.e $E^{*}=\left(x_{1}^{*} x_{2}^{*}, x_{3}^{*}, x_{4}^{*}, x_{5}^{*}, x_{6}^{*}, x_{7}^{*}, x_{8}^{*}, x_{9}^{*}\right)$.

3.2. Local Stability Analysis. In this section, we analyze the local stability of trivial and healthy equilibrium and give the condition for the existence of equilibrium of the model.

3.2.1. Local stability of trivial equilibrium. The Jacobian matrix evaluated at trivial equilibrium $E_{0}, J\left(E_{0}\right)$, is given by

$$
J\left(E_{0}\right)=\left(\begin{array}{ccccccccc}
-a_{1} & k_{2} & 0 & 0 & k_{\text {pout }} & 0 & 0 & 0 & 0 \\
b_{1} & -a_{2} & 0 & 0 & 0 & 0 & 0 & 0 & 0 \\
0 & k_{2} & -k_{c} & 0 & 0 & 0 & 0 & 0 & 0 \\
0 & 0 & k_{c} & -a_{3} & 0 & 0 & 0 & 0 & 0 \\
k_{p i n} & 0 & 0 & 0 & -a_{4} & 0 & 0 & 0 & k_{4 p} \\
0 & 0 & 0 & 0 & 0 & -\mu_{d s} & 0 & k_{4 m} & k_{4 p} \\
0 & 0 & 0 & k_{\operatorname{Ein}} & 0 & 0 & -\mu_{E} & k_{4 m} & k_{4 p} \\
0 & 0 & 0 & 0 & 0 & 0 & 0 & -a_{5} & 0 \\
0 & 0 & 0 & 0 & 0 & 0 & 0 & 0 & -a_{6}
\end{array}\right),
$$

and the eigenvalues associated to $J\left(E_{0}\right)$ are:

$\lambda_{1}=-a_{6}$,

$\lambda_{2}=-k_{c}$

$\lambda_{3}=-a_{5}$, 
$\lambda_{4}=-a_{3}$,
$\lambda_{5}=-\mu_{d s}$,
$\lambda_{6}=-\mu_{E}$,
$\lambda_{7}=-\frac{A}{3}+\frac{2^{\frac{2}{3}} Q}{6}+\frac{3^{-1} 2^{\frac{1}{3}}(H-B)}{Q}$,
$\lambda_{8}=-\frac{A}{3}-\left(\frac{2^{\frac{2}{3}} Q}{12}+\frac{3^{-1} 2^{\frac{1}{3}}(H-B)}{2 Q}\right)-\left(\frac{2^{\frac{2}{3}} 3^{\frac{1}{2}} Q}{12}-\frac{3^{-1} 2^{\frac{1}{3}}(H-B)}{2 Q}\right) i$,
$\lambda_{9}=-\frac{A}{3}-\left(\frac{2^{\frac{2}{3}} Q}{12}+\frac{3^{-1} 2^{\frac{1}{3}}(H-B)}{2 Q}\right)+\left(\frac{2^{\frac{2}{3}} 3^{\frac{1}{2}} Q}{12}-\frac{3^{-1} 2^{\frac{1}{3}}(H-B)}{2 Q}\right) i$,

where

$A=a_{1}+a_{2}+a_{4}$,

$B=a_{1} a_{2}+a_{1} a_{4}+a_{2} a_{4}$,

$C=27 a_{1} a_{2} a_{4}$,

$D=27\left(a_{4} b_{1} k_{2}+a_{2} k_{\text {pin }} k_{\text {pout }}\right)$,

$E=b_{1} k_{2}+k_{\text {pin }} k_{\text {pout }}$,

$F=a_{1}^{2}+a_{2}^{2}+a_{4}^{2}$,

$G=9 A(B-E)-2 A^{3}-C+D$,

$H=F+3 E$,

$Q=\left(G+\left(G^{2}-4(H-B)^{3}\right)^{\frac{1}{2}}\right)^{\frac{1}{3}}$,

and

$a_{1}=k_{1} R_{i b o}^{T o t}+k_{p i n}+\mu_{p}^{c}-k_{0}$,

$a_{2}=k_{2}+\mu_{T_{c}}$,

$a_{3}=k_{E i n}+\mu_{E}^{c y t}$,

$a_{4}=k_{\text {pout }}+\mu_{p}$,

$a_{5}=k_{4 m}+\mu_{I p}$,

$a_{6}=k_{4 p}+\mu_{I d s}$,

$b_{1}=k_{1} R_{i b o}^{T o t}$,

$b_{2}=k_{5} x_{7}+\mu_{d s}$,

$b_{3}=k_{5} x_{6}+k_{3} x_{5}+\mu_{E}$.

Lemma 3.2. If $Q>0$ then,

i. $E_{0}$ is locally asymptotically stable when $A>\left(2^{-\frac{1}{3}} Q+2^{\frac{1}{3}} Q^{-1}(H-B)\right)$.

ii. $E_{0}$ is a saddle point when $A<\left(2^{-\frac{1}{3}} Q+2^{\frac{1}{3}} Q^{-1}(H-B)\right)$.

Proof. Suppose $Q>0$, then:

i. $\lambda_{8}$ and $\lambda_{9}$ will have negative real parts. Also, since $a_{3}, a_{5}, a_{6}, k_{c}, \mu_{E}$, and $\mu_{d s}$ are real and positive, then the eigenvalues $\lambda_{i}, i=1,2,3, \ldots, 6$ are negative real eigenvalues.

Lastly, $\lambda_{7}$ will be negative when $A>\left(2^{-\frac{1}{3}} Q+2^{\frac{1}{3}} Q^{-1}(H-B)\right)$. Thus, $\lambda_{i}, i=$ $1,2,3,4,5,6,7,8,9$ have negative real parts, hence $E_{0}$ is locally asymptotically stable.

ii. Claiming same for $\lambda_{i}, i=1,2,3,4,5,6,8,9$. Now if $A<\left(2^{-\frac{1}{3}} Q+2^{\frac{1}{3}} Q^{-1}(H-B)\right)$ then $\lambda_{7}$ will be positive. Hence $E_{0}$ is a saddle point. 
Observe that, decreasing the rate of influx $\left(k_{0}\right)$ increases the value of A which leads to local stability of trivial equilibrium.

3.2.2. Local stability of healthy equilibrium. Using next generation method, we compute the basic replication number $\mathcal{R}_{0}$, which is the threshold parameter that measure the average number of new replicated copies generated by single virus when introduce in to a complete healthy cells over its entire period. In order to compute $\mathcal{R}_{0}$, we first write the system (2) in the form:

$$
\begin{aligned}
\frac{d X}{d t} & =\mathcal{F}(X)-\mathcal{V}(X), \\
\mathcal{V}(X) & =\mathcal{V}^{-}(X)-\mathcal{V}^{+}(X), \\
X & =\left(x_{1}, x_{2}, x_{3}, x_{4}, x_{5}, x_{6}, x_{7}, x_{8}, x_{9}\right)^{T},
\end{aligned}
$$

where $\mathcal{F}, \mathcal{V}^{+}, \mathcal{V}^{-}: \mathbf{R}^{9} \rightarrow \mathbf{R}^{9}$

Considering the infected compartments $x_{1}, x_{2}$ and $x_{5}$, then by next generation method [20, 21], the associated matrices $\mathrm{F}$ for the new infection terms, and $\mathrm{V}$ for the remaining transmission terms at healthy equilibrium are, respectively, given by

$$
F=\left(\begin{array}{ccc}
0 & 0 & 0 \\
k_{1} R_{\text {ibo }} & 0 & 0 \\
0 & 0 & 0
\end{array}\right), \quad V=\left(\begin{array}{ccc}
k_{\text {pin }}+\mu_{p}^{c y t}+k_{1} R_{\text {ibo }}-k_{0} & -k_{2} & -k_{\text {pout }} \\
0 & k_{2}+\mu_{T c} & 0 \\
-k_{\text {pin }} & 0 & k_{\text {pout }}+\mu_{p}
\end{array}\right) \text {. }
$$

Further,

$$
F V^{-1}=\left[\begin{array}{ccc}
0 & 0 & 0 \\
q & u & w \\
0 & 0 & 0
\end{array}\right]
$$

where,

$$
\begin{aligned}
q & =\frac{k_{1} R_{\text {ibo }}\left(k_{\text {pout }}+\mu_{p}\right)}{\left[\left(k_{\text {pin }}+\mu_{p}^{c y t}+k_{1} R_{\text {ibo }}-k_{0}\right)\left(k_{\text {pout }}+\mu_{p}\right)-k_{\text {pin }} k_{\text {pout }}\right]}, \\
u & =\frac{k_{2} k_{1} R_{\text {ibo }}\left(k_{\text {pout }}+\mu_{p}\right)}{\left(k_{2}+\mu_{T c}\right)\left[\left(k_{\text {pin }}+\mu_{p}^{c y t}+k_{1} R_{\text {ibo }}-k_{0}\right)\left(k_{\text {pout }}+\mu_{p}\right)-k_{\text {pin }} k_{\text {pout }}\right]}, \\
w & =\frac{k_{\text {pout }} k_{1} R_{\text {ibo }}}{\left[\left(k_{\text {pin }}+\mu_{p}^{c y t}+k_{1} R_{\text {ibo }}-k_{0}\right)\left(k_{\text {pout }}+\mu_{p}\right)-k_{\text {pin }} k_{\text {pout }}\right]} .
\end{aligned}
$$

The eigenvalues of the matrix $F V^{-1}$ are $0,0, u$. Thus, it follows that the basic replication number of the modified model is:

$$
\mathcal{R}_{0}=\rho\left(F V^{-1}\right)=\frac{k_{2} k_{1} R_{\text {ibo }}\left(k_{\text {pout }}+\mu_{p}\right)}{\left.\left(k_{2}+\mu_{T c}\right)\left[k_{\text {pin }} \mu_{p}+\left(\mu_{p}^{c y t}+k_{1} R_{\text {ibo }}\right)\left(k_{\text {pout }}+\mu_{p}\right)-\left(k_{\text {pout }}+\mu_{p}\right) k_{0}\right)\right]},
$$


where, $\rho$ is called the spectral radius, $k_{0} \geq 0$.

Lemma 3.3. $\mathcal{R}_{0}$ exists iff $k_{0}<\frac{k_{\text {pout }} \mu_{p}}{k_{\text {pout }}+\mu_{p}}+\left(\mu_{p}^{c}+k_{1} R_{i b o}\right)$.

Observe that, increasing the rate of influx $\left(k_{0}\right)$ also increases the value of $\mathcal{R}_{0}$. Likewise, decreasing $k_{0}$ decreases the value of $\mathcal{R}_{0}$. Applying Theorem 2 of [24], the following result is established.

Lemma 3.4. The healthy equilibrium $E_{1}$ of the model (2) is locally asymptotically stable if $\mathcal{R}_{0}<1$, and unstable if $\mathcal{R}_{0}>1$.

Biologically speaking, Lemma (3.4) implies that the replication can be controlled when $\mathcal{R}_{0}<1$, depending on the intial sizes of replication mechanisms in the model (2). It is noteworthy that increasing $k_{0}$ increases $\mathcal{R}_{0}$, and vice versa.

3.2.3. Threshold Analysis. In order to assess the impact of $k_{0}$, we differentiate $\mathcal{R}_{0}$ partially with respect to $k_{0}$, so we have:

$$
\frac{\partial \mathcal{R}_{0}}{\partial k_{0}}=\frac{\left(k_{\text {pout }}+\mu_{p}\right)^{2} k_{2} k_{1} R_{\text {ibo }}}{\left(k_{2}+\mu_{T c}\right)\left[k_{\text {pout }} \mu_{p}+\left(\mu_{p}^{c}+k_{1} R_{\text {ibo }}\right)\left(k_{\text {pout }}+\mu_{p}\right)-\left(k_{\text {pout }}+\mu_{p}\right) k_{0}\right]^{2}} .
$$

Since all the model parameters are non-negative, then $\frac{\partial \mathcal{R}_{0}}{\partial k_{0}}>0$, thus the following result.

Lemma 3.5. The rate of influx of HCV into Huh-7 cell have detrimental impact in the system.

\subsection{Global stability of healthy equilibrium.}

Lemma 3.6. For system (2), the healthy equilibrium $E_{1}$ is globally asymptotically stable if $\mathcal{R}_{0}<1$ and $k_{3} x_{5} x_{7}-k_{4 p} x_{9} \geq 0$.

Proof. We use the comparison theorem to prove the global stability of the healthy equilibrium $E_{1}$. The rate of change of the variables $x_{1}, x_{2}$, and $x_{5}$ of system (2) can be written as:

$$
\left(\begin{array}{l}
\frac{d x_{1}}{d t} \\
\frac{d x_{2}}{d t} \\
\frac{d x_{5}}{d t}
\end{array}\right)=(F-V)\left(\begin{array}{l}
x_{1} \\
x_{2} \\
x_{5}
\end{array}\right)-\left(\begin{array}{c}
0 \\
0 \\
k_{3} x_{5} x_{7}-k_{4 p} x_{9}
\end{array}\right)
$$

where $\mathrm{F}$ and $\mathrm{V}$ are as defined in Section 3.2. By positivity of solutions and system parameters for all $t \geq 0$, then

$$
\left(\begin{array}{l}
\frac{d x_{1}}{d t} \\
\frac{d x_{2}}{d t} \\
\frac{d x_{5}}{d t}
\end{array}\right) \leq(F-V)\left(\begin{array}{l}
x_{1} \\
x_{2} \\
x_{5}
\end{array}\right)
$$

Whenever $k_{3} x_{5} x_{7}-k_{4 p} x_{9} \geq 0$. By lemma 1 of [24] the eigenvalues of the matrix $\mathrm{F}$ - V all have negative real parts. Thus, the system (2) is stable whenever $\mathcal{R}_{0}<1$. Therefore, $\left(x_{1}, x_{2}, x_{5}\right) \rightarrow(0, \eta, 0)$ as $t \rightarrow \infty$. By the comparison theorem [24], it follows that $\left(x_{1}, x_{2}, x_{5}\right) \rightarrow(0, \eta, 0)$ and $\left(x_{3}, x_{4}, x_{6}, x_{7}, x_{8}, x_{9}\right) \rightarrow(0,0,0,0,0,0)$ as 
$t \rightarrow \infty$. Then $\left(x_{1}, x_{2}, x_{3}, x_{4}, x_{5}, x_{6}, x_{7}, x_{8}, x_{9}\right) \rightarrow E_{1}$ as $t \rightarrow \infty$. Hence, $E_{1}$ is globally asymptotically stable provided $\mathcal{R}_{0}<1$.

3.3.1. Existence of endemic equilibrium. To provide the condition for the existence of infected (endemic) equilibrium (that is, when every mechanism is present) we define the force of infection to be

$$
\lambda^{*}=k_{1} R_{i b o} x_{1}^{*} .
$$

Thus, solving system (2) at steady state gives:

$$
\begin{aligned}
x_{1}^{*} & =\frac{\phi_{2} \lambda^{*}-\phi_{1}}{\phi_{3}} \\
x_{2}^{*} & =\frac{\lambda^{*}}{k_{2}+\mu_{T c}} \\
x_{3}^{*} & =\frac{k_{2} \lambda^{*}}{k_{c}\left(k_{2}+\mu_{T c}\right)} \\
x_{4}^{*} & =\frac{k_{2} \lambda^{*}}{\left(k_{2}+\mu_{T c}\right)\left(k_{E i n}+\mu_{E}^{c}\right)} \\
x_{5}^{*} & =\left(\mathcal{R}_{0}-1\right) \frac{\alpha \psi_{1} \lambda^{*}}{\xi\left(k_{4 p}+\mu_{I d s}\right)\left(\beta_{1}-\beta_{2}\right) \rho} \\
x_{6}^{*} & =\left(\mathcal{R}_{0}-1\right) \frac{\alpha \psi_{2} \lambda^{*}}{\xi\left(k_{4 m}+\mu_{I p}\right)\left(\beta_{1}-\beta_{2}\right) \rho} \\
x_{7}^{*} & =1 \\
x_{8}^{*} & =\left(\mathcal{R}_{0}-1\right) \frac{k_{3} \alpha \psi_{1} \lambda^{*}}{\xi\left(k_{4 p}+\mu_{I d s}\right)\left(k_{4 m}+\mu_{I p}\right)\left(\beta_{1}-\beta_{2}\right) \rho} \\
x_{9}^{*} & =\left(\mathcal{R}_{0}-1\right) \frac{k_{5} \alpha \psi_{2} \lambda^{*}}{\xi\left(k_{4 m}+\mu_{I p}\right)\left(k_{4 p}+\mu_{I d s}\right)\left(\beta_{1}-\beta_{2}\right) \rho},
\end{aligned}
$$

where,

$$
\begin{aligned}
\alpha & =k_{\text {pin }} \mu_{p}+\left(\mu_{p}^{C}+k_{1} R_{\text {ibo }}-k_{0}\right)\left(k_{\text {pout }}+\mu_{p}\right) \\
\xi & =\left(k_{2}+\mu_{T c}\right) \alpha-k_{2} k_{1} R_{\text {ibo }}\left(k_{\text {pout }}+\mu_{p}\right) \\
\rho & =k_{\text {pin }}+\mu_{p}^{C}-k_{0} \\
\psi_{1} & =k_{\text {pin }} \mu_{T c}\left(\mu_{d s}\left(k_{4 p}+\mu_{I d s}\right)+k_{5} \mu_{I d s}\right) \\
\psi_{2} & =k_{3} k_{4 m} k_{\text {pin }} \mu_{T c} \\
\beta_{1} & =\frac{\left(\mu_{d s}\left(k_{4 p}+\mu_{I d s}\right)+k_{5} \mu_{I d s}\right)\left[k_{p i n}\left(k_{3}+\mu_{p}\right)-\left(k_{0}-\mu_{p}^{C}\right)\left(k_{3}+k_{p o u t}+\mu_{p}\right)\right]}{\left(k_{4 p}+\mu_{I d s}\right) \rho} \\
\beta_{2} & =\frac{k_{3} k_{4 m} k_{4 p} k_{5}}{\left(k_{4 p}+\mu_{I d s}\right)\left(k_{4 m}+\mu_{I p}\right)} \\
\phi_{1} & =\mu_{T c} \xi\left(k_{4 p}+\mu_{I d s}\right)\left(\beta_{1}-\beta_{2}\right) \rho \\
\phi_{2} & =\left(\mathcal{R}_{0}-1\right)\left(k_{2}+\mu_{T c}\right) k_{\text {pout }} \alpha \psi_{1} \\
\phi_{3} & =\xi \rho^{2}\left(k_{4 p}+\mu_{I d s}\right)\left(\beta_{1}-\beta_{2}\right) .
\end{aligned}
$$


Substituting (13) into (12) leads to

$$
a_{11} \lambda^{*}-a_{12}=0
$$

where,

$$
\begin{aligned}
& a_{11}=k_{1} R_{i b o} \phi_{2}-\phi_{3} \\
& a_{12}=k_{1} R_{i b o} \phi_{1}
\end{aligned}
$$

Hence, we have the following result.

Theorem 3.7. Suppose $\mathcal{R}_{0}>1$, then the model (2) has a unique positive endemic equilibrium if $a_{11}$ and $a_{12}$ have the same sign.

\section{NUMERICAL SIMULATIONS}

To illustrate the effect of plus-strand RNA in the model (2), we present the simulations of the model (2) using the parameter values in table 1 and at the time of transfection (i.e $t=0$ ), we assume $x_{i}(0)=0$ for $i=2,3,4,5,6,7,8,9$ and $x_{1}(0)=500$ as used by Dahari et al [2]. The numerical result is shown in figure 2 . We simulate the model (2) with different values of rate of influx $\left(k_{0}\right)$ and observe a disparity in steady state level of total plus-strand RNA, synthesized plus-strand RNA in the cytoplasm, replicated plus-strand RNA in VMS, and NS5B (which is the key replication mechanism).

\section{SENSITIVITY AND UNCERTAINTY ANALYSES}

In this section, to quantify the impact of the variations of each parameter of the model, we use partial rank correlation coefficients (PRCC) to carry out a global sensitivity analysis [28, 29]. Furthermore, an uncertainty analysis is performed, using latin hypercube sampling (LHS). The analyses are carried out for the model using the parameter values and ranges in Table 1 and the associated reproduction number as the response function. Uniform distribution is assumed for the parameters of the model and also generate 1000 LHS samples.

A box plot of the $\mathcal{R}_{0}$ as a function of the LHS runs, as depicted in Figure 4, shows that the distribution of $\mathcal{R}_{0}$ lies within the interval $\mathcal{R}_{0} \in[0.9999,1.0008]$, with a mean of $\mathcal{R}_{0}=1.0003$. Moreover, Figure 5 shows that the rate of influx, $k_{0}$ of $\mathrm{HCV}$ plus-strand RNA (which is the viral particle) is the dominant PRCC-ranked parameter for the model and then followed by the rate of production of translation complex $\left(T_{c}\right)$ and the rate of degradation, $\mu_{p}^{c y t}$, of plus-strand RNA $R_{P}^{c y t}$. Thus, these simulations suggest that when $k_{0}$ increases, then the steady state level of total plus-strand RNA in the system, synthesization plus-strand RNA in the cytoplasm, replication copies of plus-strand RNA in VMS and NS5B also increase.

\section{CONCLUSIONS}

An extension of Dahari et al [2] model of sub-genomic HCV replication in Huh-7 cell is presented. The qualitative analysis shows that the model has three equilibria viz trivial equilibrium, healthy equilibrium and endemic equilibrium. The 
trivial equilibrium could either be locally asymptotically stable or saddle point depending on certain threshold quantity. Basic replication number is obtained and found that the healthy equilibrium $E_{1}$ is globally asymptotically stable when $\mathcal{R}_{0}<1$ and unique positive endemic equilibrium exists when $\mathcal{R}_{0}>1$. Further, threshold analysis on $\mathcal{R}_{0}$ reveals that high rate of influx of plus-strand RNA can cause detrimental effect. It is shown that changing the rate of influx changes the steady state level of total plus-strand RNA in the system, synthesizes plus-strand RNA in the cytoplasm, replicates plus-strand RNA in VMS and NS5B (which is the key replication mechanism). It is observed, as depicted in Figure 3, that $\mathcal{R}_{0}$ is directly proportional to the rate of influx. Sensitivity and uncertainty analyses of the model (using the basic replication number $\left(\mathcal{R}_{0}\right)$ as the response function) show that the top three PRCC-ranked parameters are the rate of influx $\left(k_{0}\right)$ of $\mathrm{HCV}$ plus-strand RNA, the rate of production of translation complex $\left(T_{c}\right)$ and the rate of degradation $\left(\mu_{p}^{c y t}\right)$ of plus-strand RNA $R_{P}^{c y t}$. Furthermore, the distribution of $\mathcal{R}_{0}$ is between $[0.9999,1.0008]$ with a mean of $\mathcal{R}_{0}=1.0003$.

\section{REFERENCES}

[1] Philippe R., Christophe H., Emmanuelle B., Denys B. and Malika A., Hepatitis C virus ultra-structure and morphogenesis, Biology of the cell, 96(2004), 103-108.

[2] Harel D., Ruy M. R., Charles M. R. and Alan S. P., Mathematical Modeling of Subgenomic Hepatitis C Virus Replication in Huh-7 Cells, Journal of Virology, 81(2007), 750-760.

[3] Nurgary S., Mathematical Modeling of Hepatitis C Virus Replication, Heidelberg University, 2012

[4] Spiegelman P., Extracellular evolution of replicating molecules, The neurosciences, Rockefeller university, New York, In F. O. Schmitt (ed.)(1970), 927.

[5] Wasley A. and Alter M. J., Epidemiology of Hepatitis C: geographic differences and temporal trends, Semin liver Dis., 20(1),(2000) 1-16.

[6] Lohmann V., Korner F., Koch J., Herian U., Theilmann L. and Bartenschlager R., Replication of subgenomic hepatitis C Virus RNA in a hepatoma cell line,Science, 285(1999), 110-113.

[7] Bartenschlager R., Frase M. and Pietschmann T., Novel insights into hepatitis C virus replication and persistence, Advances in virus research, Academic press(2004), 71-180.

[8] Quinkert D., Bartenschlager R. and Lohmann V., Quantitative Analysis of the Hepatitis C Virus Replication Complex, Journal of Virology, 79(21)2005, 13594-13605.

[9] Ashfaq U. A., Javed T., Rehman S., Nawaz Z. and Riazuddin S., An overview of HCV molecular biology, replication and immune response, Virology J., 8(161) 2011.

[10] Dubuisson J., Helle F. and Cocquerel L., Early steps of the hepatitis C virus life cycle, Cellular Microbiology, 10(4)2008, 821-827.

[11] Popescu C. and Dubuisson J., Role of lipid metabolism in hepatitis C virus assembly and entry, Biol. Cell, 102(2010) 63-74.

[12] Burlone M. E. and Budkowska, Hepatitis C virus entry: role of lopoprotein and cellular receptor, J. of General Virology, 90(2009), 1055-1070.

[13] Ma H., Leveque V., Witte D. A., Li W., Hendricks T., Clausen S. M., Cammack N. and Klumpp K., Inhibition of native hepatitis $\mathrm{C}$ virus replicase by nucleotide and non-nucleoside inhibitors, Virology 332(2005), 8-15.

[14] Chatterjee A., Guedj J. and Perelson A. S., Review Mathematical modelling of HCV infection: what can it teach us in the era of direct-acting antiviral agents?, Antivaral Therapy, 17(2012),1171-1182.

[15] Bartenschlager R. and Lohmann V., Replication of hepatitis C virus, Journal of General Virology, 81(2000), 1631-1648. 
[16] Lavanchy D., Purcell R., Hollinger F. B., Howard C., Alberti A., Kew M., Dusheiko G., Alter M., Ayoola E., Beutels P., Bloomer R., Ferret B., Decker R., Esteban R., Fay O., Fields H., Fuller E. C., Grob P., Houghton M., Leung N., Locarnini S. A., Margolis H., Meheus A., Miyamura T., Mohamed M. K., Tandon B., Thomas D., Head H. T., Toukan A. U., Van D. P., Zanetti A., Arthur R., Couper M., D’Amelio R., Emmanuel J. C., Esteves K., Gavinio P., Griffiths E., Hallaj Z., Heuck C. C., Heymann D. L., Holck S. E., Kane M., Martinez L. J., Meslin F., Mochny I. S., Ndikuyeze A., Padilla A. M., Rodier G. M., Roure C., Savage F. and Vercauteren G., Global surveillance and control of hepatitis C, Journal of Viral Hepatitis, 6(1999), 35-47.

[17] Stuyver J. L., McBrayer R. T., Tharnish M. P., Hassan E. A., Chu K. C., Pankiewicz W. K., Watanabe A. K., Schinazi F. R. and Otto J. M., Dynamics of Subgenomic Hepatitis C Virus Replicon RNA Levels in Huh-7 Cells after Exposure to Nucleoside Antimetabolites, Journal of Virology, 77(19)2003, 10689-10694.

[18] Eigen M., Biebricher C. K., Gebinoga M. and Gardiner W. C., The hypercycle Coupling of RNA and protein biosynthesis in the infection cycle of an RNA bacteriophage, Biochemistry,30(46)1991, 11005-11018.

[19] Biebricher C. K., Eigen M. and Luce R., Kinetic analysis of template-instructed and de novo RNA synthesis by Q $\beta$ replicase, J. Mol. Biol., 148(1981), 391-410.

[20] Nafiu H., Winter M., and A. B. Gumel, Qualitative Assessment of the Role of Public Health Education Program on HIV Transmission Dynamics, Mathematical Medicine and Biology, 28(2011), 245-270.

[21] Van den Driessche P. and Watmough J., Reproduction Numbers and Sub-Threshold Endemic Equilibria for Compartmental Models of Disease Transmission, Math. Biosci., 180(2002), 2948.

[22] Chu P. W. and Westaway E. G., Replication strategy of Kunjin virus:evidence for recycling role of replicative form RNA as template in semiconservative and asymmetric replication, Virology, 140(1985), 68-79.

[23] Dahari H., Feliu A., Garcia-Retortillo M., Forns X. and Neumann A. U., Second hepatitis C replication compartment indicated by viral dynamics during liver transplantation, J. Hepatol, 42(2005), 491-498.

[24] Lakshmikantham V. , Leela S. , and Martynyuk A. A., Stability Analysis of Nonlinear Systems, vol. 125, Marcel Dekker, New York, Ny, USA, 1989.

[25] Dahari H, Feinstone SM, Major ME, Meta-analysis of hepatitis C virus vaccine efficacy in chimpanzees indicates an importance for structural proteins. Gastroenterology, 139(2010), 965-974.

[26] Nikita V. I., Elena L. M., Ilya R. A., Pavel S. D., Vitaly A. L., Konstantin N. K., Dmitry I. T., Vitaly V. G., Maria G. S., Alexander M. S., Diana C., Lars K., Nikolay A. K., Vladimir A. I., A New Stochastic Model for Subgenomic Hepatitis C Virus Replication Considers Drug Resistant Mutants, Plos One Journal, 9(3)2014, 1-17.

[27] Hai-Feng H. and Na-Na S., Global Stability for a Binge Drinking Model with Two Stages, Discrete Dynamics in Nature and Society,2012, 1-15.

[28] Blower, S.M. Dowlatabadi, H., Sensitivity and uncertainty analysis of complex models of disease transmission: an HIV model, as an example, International Statistical Review 2 (1994) 229-243.

[29] Hussaini, N., Lubuma, J. M-S., Barley K. and Gumel, A.B.: Mathematical analysis of a model for AVL-HIV co-endemicity. Mathematical Biosciences. 271 (2016): 80-95.

[30] WHO, World Health Organisation, Hepatitis C FactSheet, accessed 10th March, 2019. www.who.intnews-roomfact-sheetsdetailhepatitis-c. 
A

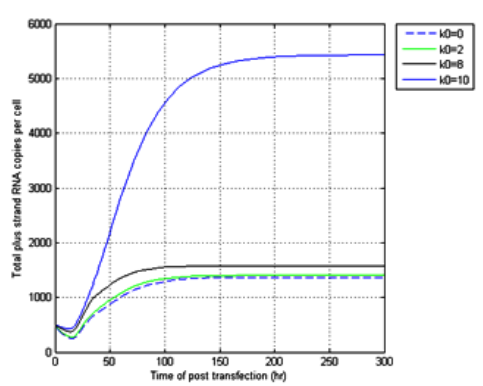

C

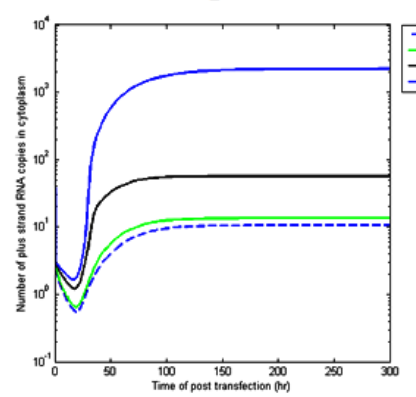

B

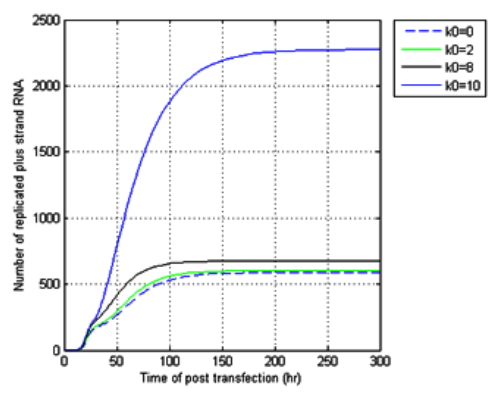

D

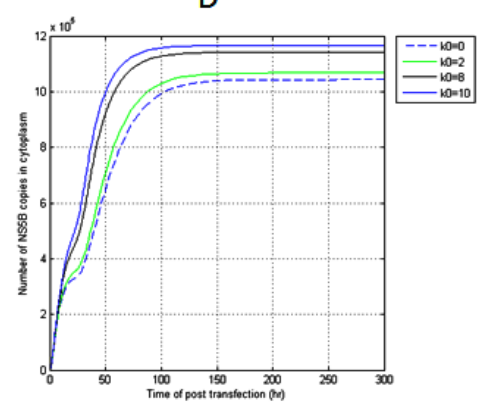

Figure 2. Simulations of the model (2) showing the disparity in steady state level of (A) Total plus-strand RNA in the system, (B) Synthesized plus-strand RNA in the cytoplasm, (C)Replicated plus-strand RNA in VMS, and (D) NS5B (which is the key replication mechanism). 


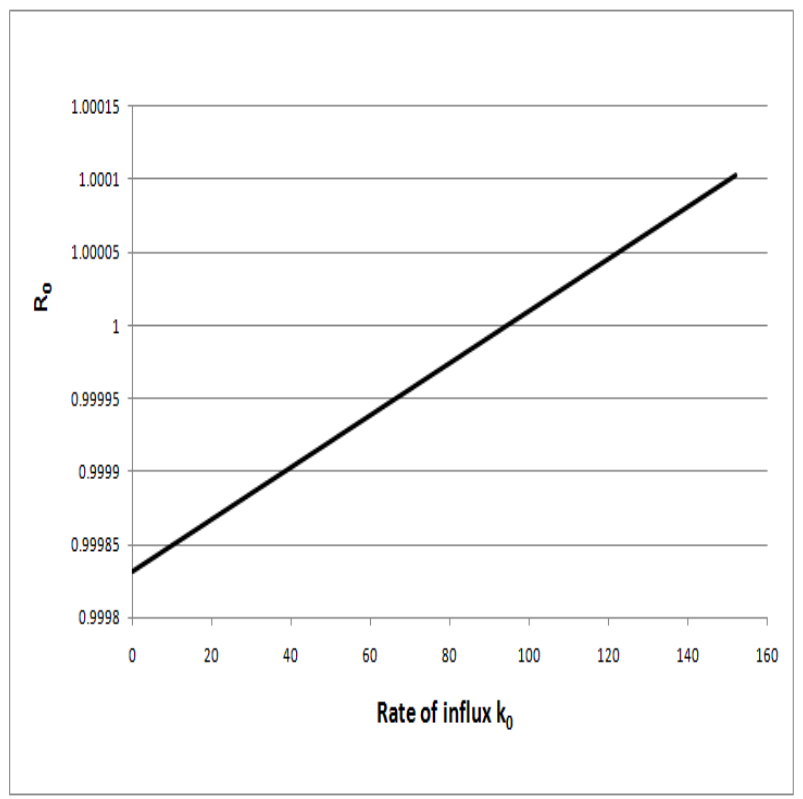

FIGURE 3. Graph showing increase in $\mathcal{R}_{0}$ due to increase in rate of influx 


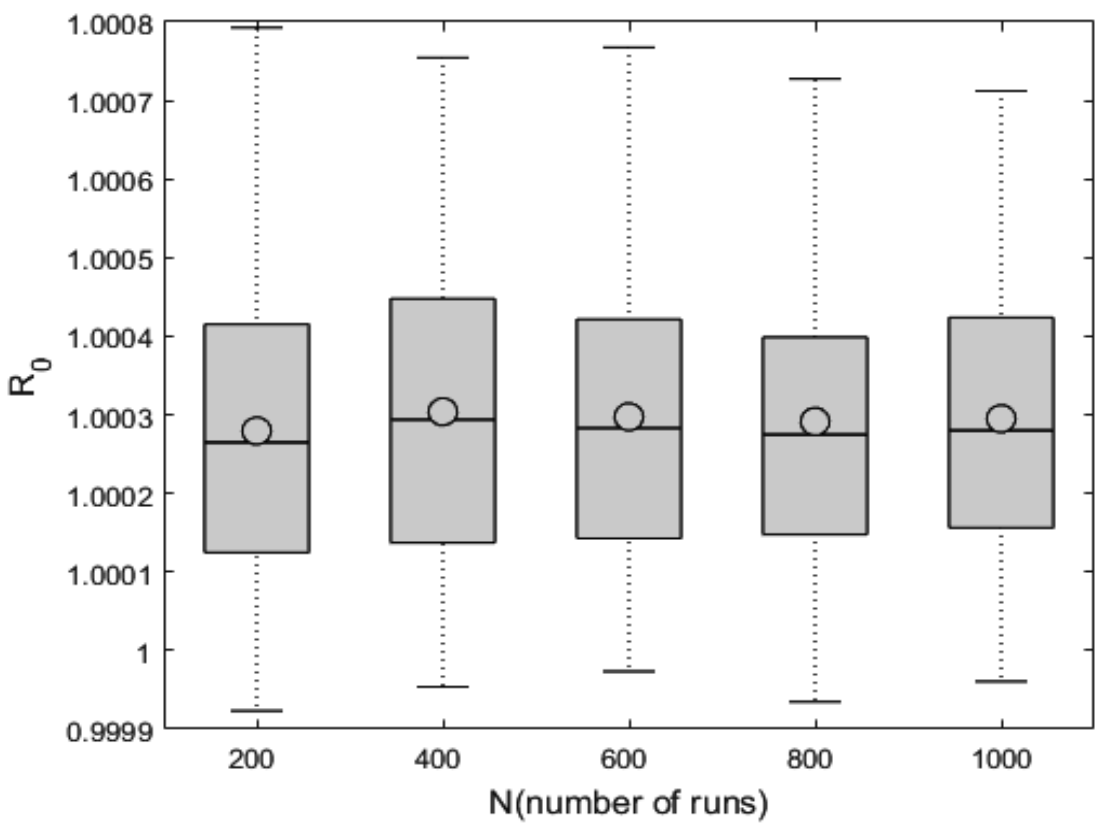

Figure 4. Box plot of $\mathcal{R}_{0}$ as a function of the LHS runs. Parameter values used are as in Table 1. 


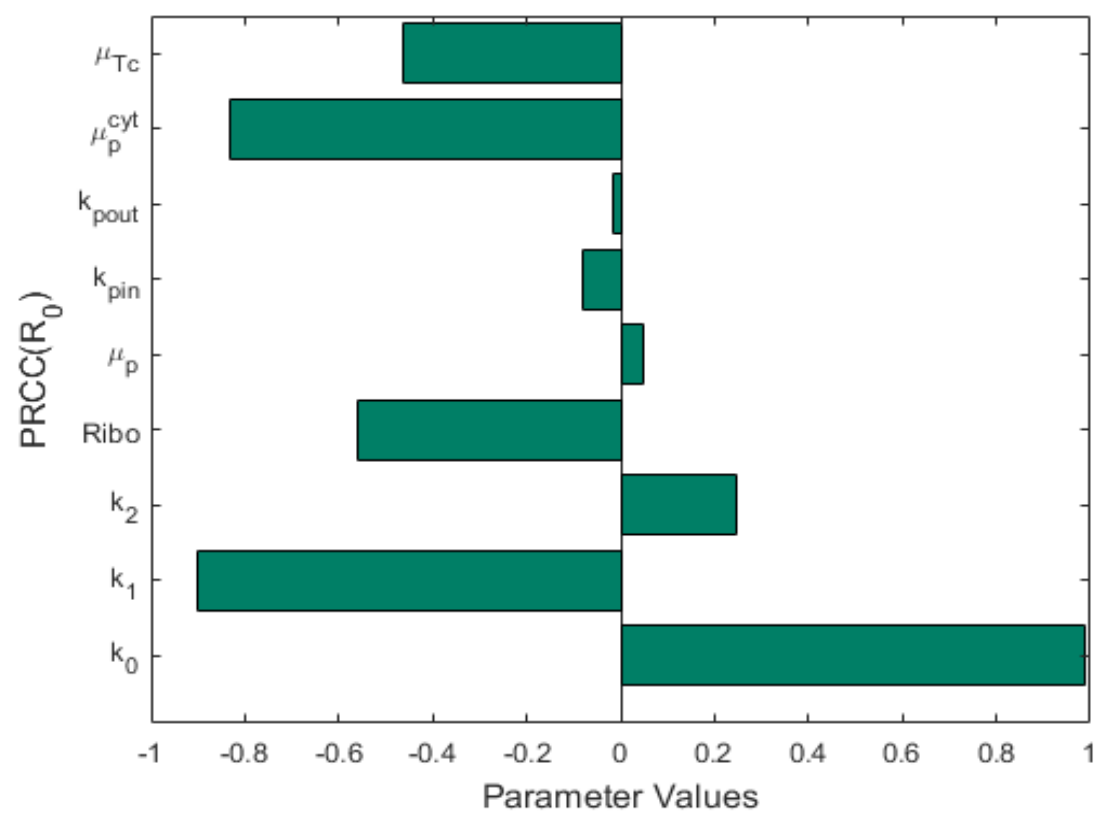

Figure 5. Distribution of PRCC values for the model (2) using $\mathcal{R}_{0}$ as the response function. Parameters and ranges used are as in Table 1. 